\title{
Parasellar extra-axial cavernoma mimicking meningioma: a case report
}

\author{
Arun Oommen ${ }^{1}$, Thara Pratap ${ }^{2}$, Sushil Chandi ${ }^{3}$, Muhammed Jasim Abdul Jalal ${ }^{4}$ \\ ${ }^{1}$ Department of Neurosurgery, VPS Lakeshore Hospital, Kochi, Kerala 682040, India. \\ ${ }^{2}$ Department of Radiology, VPS Lakeshore Hospital, Kochi, Kerala 682040, India. \\ ${ }^{3}$ Department of Pathology, Lisie Hospital, Kochi, Kerala 682040, India. \\ ${ }^{4}$ Department of Internal Medicine and Rheumatology, VPS Lakeshore Hospital, Kochi, Kerala 682040, India.
}

Correspondence to: Dr. Muhammed Jasim Abdul Jalal, Department of Internal Medicine and Rheumatology, VPS Lakeshore Hospital, Nettoor.P.O., Maradu, NH 47-Byepass, Kochi, Kerala 682040, India. E-mail: jasimabduljalal@yahoo.com

How to cite this article: Oommen A, Pratap T, Chandi S, Jalal MJA. Parasellar extra-axial cavernoma mimicking meningioma: a case report. Neuroimmunol Neuroinflammation 2017;4:16-9.

Article history:

Received: 24-11-2016

Accepted: 20-12-2016

Published: 20-01-2017

Key words:

Extra-axial cavernoma, parasellar cavernoma, meningioma

\section{ABSTRACT}

Parasellar extra-axial cavernomas are rare lesions. The authors report a case of extra-axial cavernoma in a 50-year-old male patient, who presented with occipital headache and double vision. The magnetic resonance imaging showed an enhancing extra-axial dural-based mass in the left parasellar region invading cavernous sinus, hyper-intense on T2-weighted images, iso-intense on T1-weighted images and high relative cerebral blood velocity on magnetic resonance perfusion. The patient underwent a left pterional craniotomy and parasellar space occupying lesion was excised. Histopathology was suggestive of cavernous hemangioma.

\section{INTRODUCTION}

Cavernomas are benign. They usually have an intraparenchymal origin, but occasionally arise from the duramater. Extra-axial cavernous angiomas account for $0.4-2 \%$ of all intracranial vascular malformations. They usually occur in the middle cranial fossa, associated with the cavernous sinus. Here, we report a case of a 50-year-old male patient who presented with left occipital headache and diplopia due to a parasellar space occupying lesion mimicking a meningioma.

\section{CASE REPORT}

\section{Case history}

A 50-year-old man had a 1 month history of left occipital headache unresponsive to drug therapy with common analgesics. The intensity of this symptom increased over time. Later, it was associated with double vision. There was no history of seizures, vomiting and limb weakness. The patient was hemodynamically stable. His neurological examination was normal, except for left 6th and partial left 3rd nerve palsy. 


\section{Imaging}

The magnetic resonance imaging (MRI) showed an enhancing extra-axial dural-based mass in the left parasellar region invading cavernous sinus; the lesion size was $5.7 \mathrm{~cm} \times 3.8 \mathrm{~cm}$. It was hyper-intense on T2weighted images [Figure $1 \mathrm{~A}$ ] with flow voids and isointense on T1-weighted images [Figure 1B]. There was marked homogenous enhancement in post-contrast study [Figure 1C]. MR perfusion showed high relative cerebral blood velocity [Figure 1D].

\section{Surgery}

The patient underwent a left extended pterional craniotomy with extradural clinoidectomy. Thorough devascularization of the extradural tumor based blood vessels was done. Dura was opened based on the middle meningeal artery and anterior sylvian fissure splitting was done. Enucleation and tumor debulking was done using ultrasonic aspirator, suction and bipolar cautery under microscopic assistance. Tumor was stiff, highly vascular, greyish white to red in colour and there was a clear arachnoid plane between the space occupying lesion and brain parenchyma. It was dura based on greater wing of sphenoid and left parasellar area. Excision of the tumor was almost total. The middle cerebral artery branches, 2nd, 3rd, 4th and 6 th cranial nerves were identified and preserved.

\section{Histopathology}

Histopathology showed sections of a tumor mass composed of compactly aggregated vascular channels of varied sizes with thick hyalin walls and lined with endothelial cells. There were occasional foci of calcifications. But there were no other tissue element including meningothelial cells around the tumor mass or among the vascular channels. The findings were
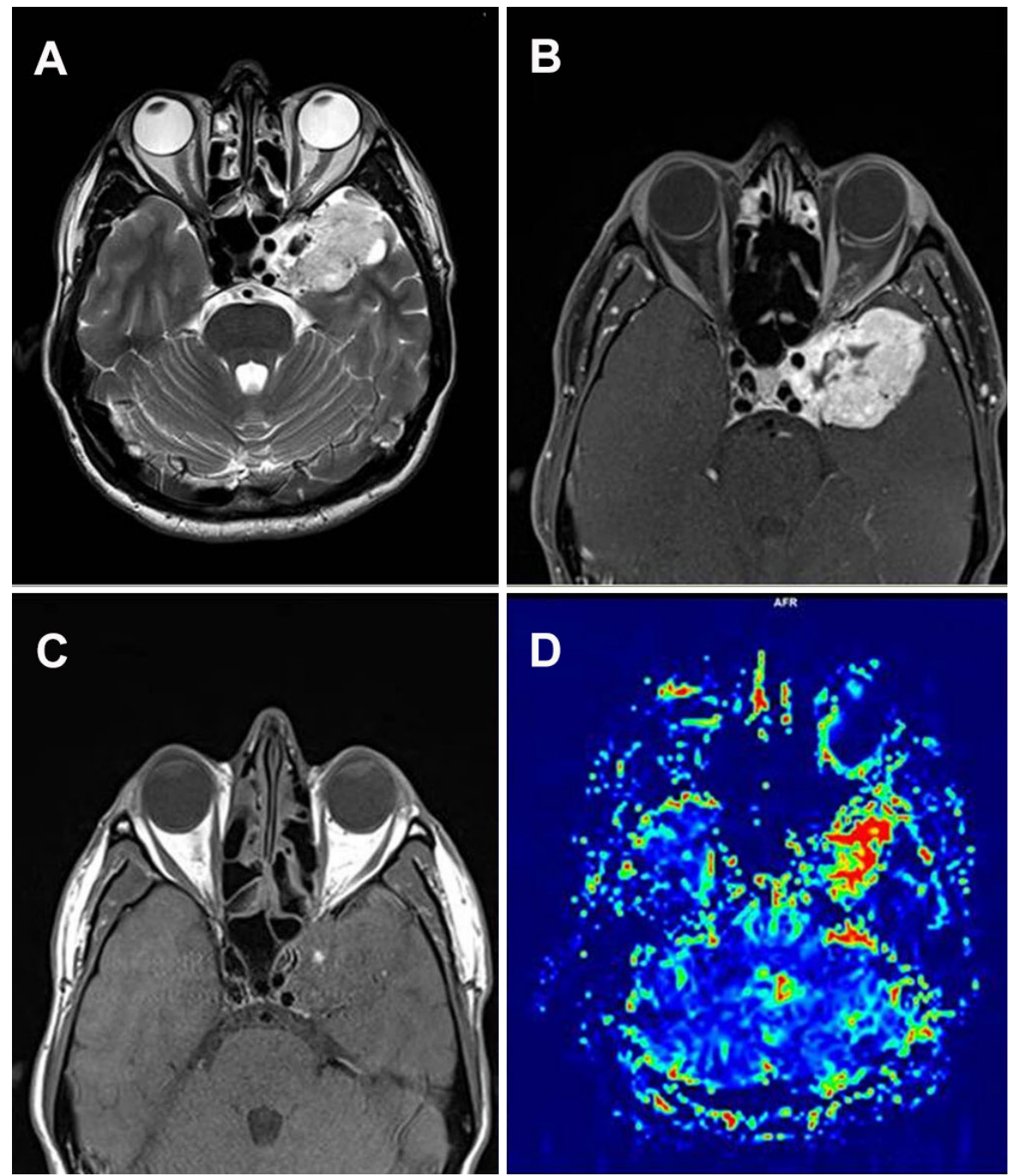

Figure 1: (A) High-resolution Axial T2 Fat sat sequences demonstrate hyperintense extra-axial lesion located in the left parasellar region, which extends to middle cranial fossa. The lesion partly encircles left distal ICA; (B) axial T1 W sequence shows iso-intense lesion; (C) post-contrast Axial T1 W sequences show marked homogenous enhancement; (D) perfusion map of CBV was obtained. High rCBV noted, demonstrating a highly vascular lesion. CBV: cerebral blood volume 
suggestive of cavernous hemangioma [Figure 2].

\section{Follow-up}

The postoperative MRI brain with contrast did not show any residual hemangioma [Figure 3]. The patient was asymptomatic without any additional neurologic deficit.

\section{DISCUSSION}

Cavernous angiomas are vascular malformations. They are composed of enlarged sinusoidal vessels arranged in clusters, enclosed by a thin endothelial wall without interposed tissue within. They lack an elastic lamina, smooth muscles and are sometimes ossified or calcified. The term "cavernous angioma" has been used interchangeably with "cavernous hemangioma," "cavernous malformation," or "cavernoma". These lesions are vascular abnormalities rather than neoplastic processes. ${ }^{[1]}$

Cavernomas most commonly originates from the brain parenchyma. However, they also arise intraspinally, or from the dura. ${ }^{[2]}$ Extra-axial dural-based cavernous malformations are extremely rare when compared to their intra-axial counterparts. In 1994, Lewis et al. ${ }^{[3]}$ identified two types of dural cavernous angiomas.

(1) One present in the dura of the middle cranial fossa usually in the vicinity of the cavernous sinus.

(2) The second type consists of dural-based lesions such as the convexity, cerebral and cerebellar falx, the tentorium, posterior fossa, and the floor of the anterior fossa. ${ }^{[4]}$

The separation of the two types is important due to the differences in the patient population affected and the more aggressive clinical course of dural-based cavernous angiomas in the middle cranial fossa.

The malformations of the first group are more clinically aggressive because of their localization and vascular supply. In these cases, both preoperative radiation and embolization are recommended because they reduce intraoperative bleeding risk.

On the other hand, in the cases of the second group, neither radiation nor embolization is necessary to successfully remove cavernous hemangiomas outside the middle cranial fossa, since their vascular supply can be easily controlled through the surgical exposure..$^{[5]}$
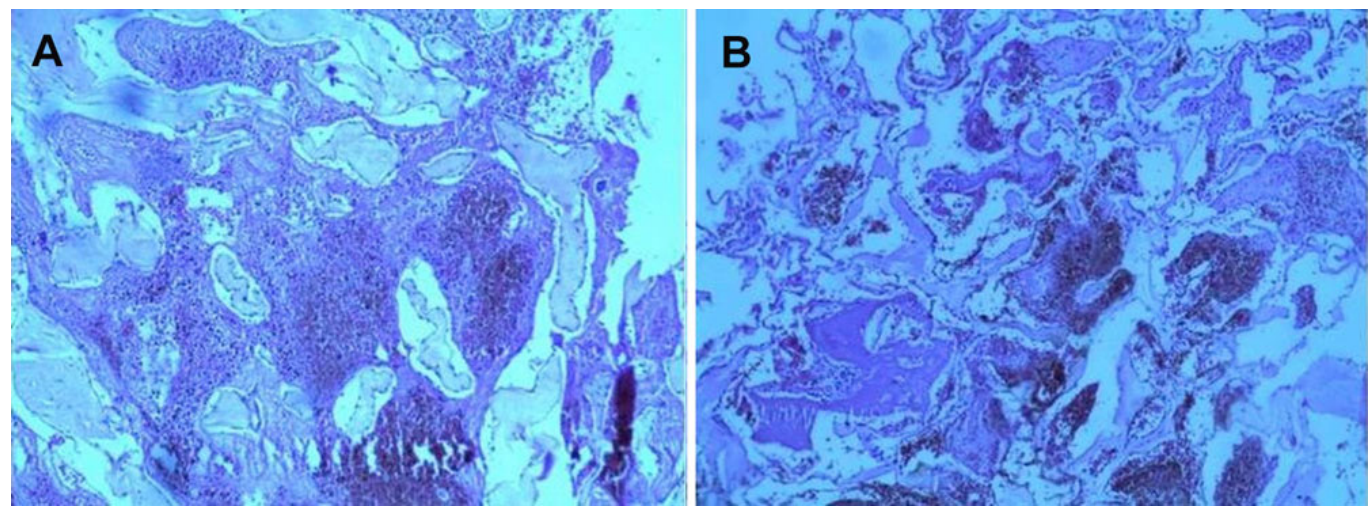

Figure 2: HE staining at low magnification $(\times 10)$ showing $(A)$ cavernous haemangioma and $(B)$ dilated vascular channels with fibrous walls, devoid of intervening neuroglial tissue


Figure 3: Postoperative magnetic resonance images. (A) Axial T2W sequences show fluid signal collection in the left parasellar region; (B) axial FLAIR reveals suppression of fluid signal; (C) axial post-contrast T1 shows no contrast enhancement, suggesting no residual lesion 
Dural-based cavernomas are histologically similar to intraparenchymal cavernous angiomas. However, Rosso et al. ${ }^{[6]}$ showed a completely different appearance on computed tomography (CT), MRI and angiography. This fact makes accurate preoperative diagnosis very difficult, as imaging findings can be varied and resemble meningiomas.

Cavernous dural hemangiomas can closely resemble meningiomas on CT and MRI in terms of signal characteristics, enhancement pattern, and localization.

The CT appearance of dural cavernous hemangiomas, like meningiomas, shows a well-defined extra-axial mass lesion on a broad dural base with regular contrast. ${ }^{[5]}$ Occasional calcifications may be present. ${ }^{[5]}$ The cerebral angiography may not be performed when CT or MRI findings are felt to be diagnostic, and the lesion is surgically accessible..$^{[5]}$

On MRI, cavernomas are usually either isointense or hypointense on T1 sequences and mixed to hyperintense on T2W sequences. Much like on CT, they also usually enhance on MRI. Meningiomas can also appear hyper-intense on T2 sequences, though they are usually hypo-intense. Hence, a T2 hyperintense lesion is far from specific for dural-based cavernoma outside the middle cranial fossa. Moreover, cavernomas can have dural tails like meningiomas, and may also cause significant perilesional edema. ${ }^{[7]}$

Angiography can show a tumor blush, hypervascularity or an avascular mass. Even angiography can be completely negative, or demonstrate pooling of contrast medium during the late venous phase.

In the present scenario, where medical and surgical sciences have advanced, stereotactic radiosurgery represents a therapeutic option. This seems to be crucial because dural cavernous hemangiomas are different from meningiomas in their clinical features such as surgical difficulty and sensitivity to radiosurgery. Although the treatment of choice is the total surgical removal, it is also possible to treat the lesion with radiosurgery after the histopathological confirmation in cases of a partial removal.

In conclusion, the occurrence of dural cavernomas may be suspected in the presence of extra-axial parasellar space occupying lesions especially if neuroradiological data suggest a meningioma. Intracavernous hemangioma give high signal intensity on $\mathrm{T} 2 \mathrm{~W}$ images, with a strong homogenous enhancement on contrast enhanced T1W images. It is important to keep this entity among the differentials when planning resection of a lesion that seems to be a meningioma. Cavernous angiomas bleed profusely during surgery, hence perioperative mortality is high as a result of uncontrollable bleeding. Preoperative angiogram and embolization are also recommended. The treatment of choice should always include surgery since it allows histopathological confirmation.

\section{Financial support and sponsorship \\ Nil.}

\section{Conflicts of interest}

There are no conflicts of interest.

\section{Patient consent}

Informed consent was obtained from the patient.

\section{Ethics approval}

The patient was treated within the standards of our institute and the report was approved.

\section{REFERENCES}

1. Kashlan ON, Sack JA, Ramnath S. Cavernous angioma of the dural convexity mimicking a meningioma. Austin Neurosurg Open Access 2014;1:1019.

2. Riant F, Bergametti F, Fournier HD, Chapon F, Michalak-Provost S, Cecillon M, Lejeune P, Hosseini H, Choe C, Orth M, Bernreuther C, Boulday G, Denier C, Labauge P, Tournier-Lasserve E. CCM3 mutations are associated with early-onset cerebral hemorrhage and multiple meningiomas. Mol Syndromol 2013;4:165-72.

3. Lewis AI, Tew JM Jr, Payner TD, Yeh HS. Dural cavernous angiomas outside the middle cranial fossa: a report of two cases. Neurosurgery 1994;35:498-504.

4. Tsutsumi S, Yasumoto Y, Saeki H, Ito M. Cranial dural cavernous angioma. Clin Neuroradiol 2014;24:155-9.

5. Di Vitantonio H, De Paulis D, Ricci A, Marzi S, Dehcordi SR, Galzio RJ. Cavernous hemangioma of the dura mater mimicking meningioma. Surg Neurol Int 2015;6:S375-8.

6. Rosso D, Lee DH, Ferguson GG, Tailor C, Iskander S, Hammond RR. Dural cavernous angioma: a preoperative diagnostic challenge. Can J Neurol Sci 2003;30:272-7.

7. Joshi V, Muzumdar D, Dange N, Goel A. Supratentorial convexity dural-based cavernous hemangioma mimicking a meningioma in a child. Pediatr Neurosurg 2009;45:141-5. 